\title{
Trasplante de microbiota fecal: una revisión
}

\author{
Fecal Microbial Transplantation: A Review
}

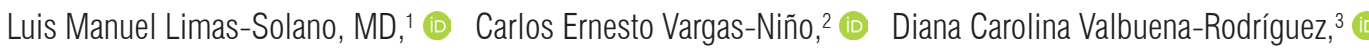 \\ Laura Ximena Ramírez-López. ${ }^{*}$
}

\author{
Gacceso abierto \\ Citación: \\ Limas-Solano LM, Vargas-Niño CE, Valbuena- \\ Rodríguez DC, Ramírez-López LX. Trasplante \\ de microbiota fecal: una revisión. Rev Colomb \\ Gastroenterol. 2020;35(3):329-337. https://doi. \\ org/10.22516/25007440.486
}

Médico, especialista en Cirugía General y Gastroenterólogo Clínico Quirúrgico; Tunja, Boyacá (Colombia).

2 Estudiante de Bacteriología y Laboratorio Clínico, Universidad de Boyacá; Tunja, Boyacá (Colombia).

3 Bacterióloga, Magíster en Salud Pública,

Universidad de Boyacá; Tunja, Boyacá

(Colombia).

4 Bacterióloga, Magíster en Bioquímica Clínica,

Universidad de Boyacá; Tunja, Boyacá

(Colombia).

*Correspondencia:

Laura Ximena Ramírez-López, MD

lauramirez@uniboyaca.edu.co

Fecha recibido: $\quad 25 / 11 / 19$

Fecha aceptado: 28/02/20

\section{Resumen}

La microbiota intestinal sana se define a partir de la presencia de grupos de microorganismos que potencian el metabolismo del huésped. Estos microorganismos le confieren resistencia ante las infecciones, así como ante procesos inflamatorios y frente al desarrollo de neoplasias o autoinmunidad. Además, favorecen las funciones endocrinas y colaboran con la función neurológica a través del eje intestino-cerebro. Por otro lado, el trasplante de microbiota fecal consiste en la introducción de una suspensión de materia fecal de un donante sano en el tracto gastrointestinal de otra persona, que generalmente es un paciente que presenta una patología concreta. Esto se realiza con el fin de manipular la composición de la microbiota del destinatario y contribuir al tratamiento de su problema. El concepto de trasplante de microbiota fecal rompe con la consideración tradicional de las bacterias como elementos dañinos y presta atención a las que, probablemente, son las más subvaloradas de las excretas del cuerpo humano: las heces. En efecto, se ha evidenciado su alta eficacia y el procedimiento es reconocido por el número de pacientes a los que ha ayudado, que se puede ya cifrar en miles. El objetivo de esta revisión de literatura fue describir aspectos básicos para comprender el trasplante de microbiota fecal enfocado al tratamiento de infecciones producidas por Clostridioides difficile.

\section{Palabras clave}

Disbiosis, microbiota fecal, trasplante de microbiota fecal, bacterioterapia, infección, Clostridium difficile.

\section{Abstract}

Gut microbiota is defined as healthy when there are groups of microorganisms that enhance the host's metabolism, confer resistance to infections, inflammatory processes, the development of malignancies or autoimmunity, promote endocrine functions and support neurological function through the so-called gut-brain axis. Fecal microbial transplantation is the transfer of fecal matter from a healthy donor into the gastrointestinal tract of another person, usually a patient with a specific pathology, to manipulate the composition of the recipient's microbiota and contribute to the treatment of his or her condition. The concept of fecal microbial transplantation breaks with the traditional thought of bacteria as harmful elements and draws attention to what is probably the most undervalued of the human body's excreta: feces. Its high efficiency has been demonstrated and the procedure is recognized by the many patients it has helped, which can already be counted in thousands. The objective of this literature review was to describe the basics of fecal microbial transplantation for the treatment of Clostridioides difficile infections.

\section{Keywords}

Dysbiosis; Fecal microbiota; Fecal microbial transplantation; Bacteriotherapy; Infectionn; Clostridium difficile. 


\section{INTRODUCCIÓN}

La microbiota más abundante y mejor estudiada en el cuerpo humano reside en el tracto intestinal. Su impacto extiende los límites de la interfaz mucosa, ya que desempeña un papel esencial en las funciones sistémicas, como el desarrollo del sistema inmunitario (1). Antes que apareciera la penicilina en la década de los 40, las enfermedades infecciosas eran la principal causa de muerte del ser humano, y lo siguen siendo en gran parte del mundo. En efecto, se ha visto un aumento significativo de la resistencia bacteriana a los antimicrobianos, lo cual ha suscitado una gran preocupación, dado que esto constituye un obstáculo para el tratamiento de agentes infecciosos, pero también genera un gran interés respecto al desarrollo de nuevas estrategias terapéuticas (2).

Una de estas estrategias es el trasplante de microbiota fecal (TMF), el cual se define como la introducción de una suspensión de microbiota fecal, debidamente procesada y derivada de un donante sano, que es inoculada en el tracto gastrointestinal de un individuo enfermo. En vista de que este no es un concepto terapéutico, en los últimos años el TMF ha generado gran interés, pues se ha convertido en la terapia de elección para el tratamiento de infecciones causadas por Clostridioides difficile (anteriormente denominada Clostridium difficile) (3). Así, el TMF ya no se considera una práctica médica alternativa, sino que ha ganado la aceptación como una terapia valiosa en la actualidad, aunque todavía es poco conocida a nivel mundial. Su popularidad se ha acrecentado por su facilidad de uso, factibilidad y eficacia (4).

\section{METODOLOGÍA}

Para el desarrollo de la presente revisión de tema, se realizó una búsqueda de la literatura utilizando los siguientes términos DeCS (Descriptores en Ciencias de la Salud) y MeSH (Medical Subject Headings), así como palabras clave en la estrategia de búsqueda: disbiosis, microbiota fecal, trasplante de microbiota fecal, bacterioterapia, infección, Clostridium difficile.

La búsqueda se limitó a estudios elaborados en humanos, en idiomas inglés, francés y español, publicados desde 2013 hasta el presente. Las bases de datos electrónicas investigadas fueron Science Direct, Redalyc, PubMed y NCBI. Además, se realizaron búsquedas manuales en bases de datos para literatura gris y Google Académico. Se eligieron las publicaciones más relevantes según los autores.

\section{MICROBIOTA INTESTINAL}

La microbiota intestinal consta de numerosas bacterias, virus y hongos que viven en el contenido intestinal (masa fecal), así como en el moco que cubre la mucosa intestinal. Estos dos hábitats constituyen dos ecosistemas comensales microbiológicos separados, que tienen diferentes funciones en la interferencia entre ellos y el organismo del huésped. En la actualidad, se han identificado más de 1000 géneros de bacterias intestinales (5).

En estados de salud, la microbiota intestinal se ha compuesto de cuatro familias de bacterias: Bacteroidetes, Firmicutes y, en menor medida, Proteobacteria y Actinobacteria (6). La exploración de este ecosistema, que coexiste con el organismo humano, ha sido posible con la introducción de técnicas de investigación de $\mathrm{ADN}$ molecular, con la secuenciación de ARN ribosómico 16S (ARNr) (7). Esto se da en virtud de que los métodos microbiológicos clásicos utilizados para identificar cepas de bacterias u hongos, como el cultivo microbiológico, son ineficaces en el caso de la microbiota humana, ya que algunas cepas bacterianas del intestino no pueden cultivarse en condiciones de laboratorio.

Así pues, el número de células bacterianas existentes en el tracto digestivo humano del individuo sano alcanza los 100 billones, 10 veces más que el de células del cuerpo humano (5). Por tanto, la microbiota intestinal desempeña un papel clave en la salud y se reconoce cada vez más como un contribuyente a varios estados de enfermedad, cuando se produce un desequilibrio (8) llamado disbiosis, y, en comparación con la microbiota de controles sanos, tiene una menor abundancia de bifidobacterias y una mayor abundancia de bacterias gramnegativas (9). Estas últimas tienen múltiples funciones que involucran el sistema inmunitario de mucosas y la resistencia a la colonización contra, por ejemplo, el Clostridioides difficile (10).

\section{TMF E INFECCIÓN POR CLOSTRIDIOIDES DIFFICILE (ICD)}

La microbiota es considerada un tejido y en el TMF se utiliza para implantar la suspensión de microbiota fecal de un donante sano en el tracto gastrointestinal de una persona enferma o un receptor (11). Esto, con el objetivo de recuperar la composición microbiana (12). Dicho proceso, además, mejora el estado disbiótico, mediante el aumento de la diversidad general y la restauración de la funcionalidad de la microbiota (3).

Con este panorama, el TMF se está usando cada vez más para tratar la ICD, dado que el $C$. difficile es un bacilo grampositivo, anaeróbico, formador de esporas, asociado con infecciones endógenas (colonización) o exógenas (infecciones asociadas a la atención en salud / consumo o indicación de antibióticos de amplio espectro). La patogénesis se atribuye, principalmente, a las toxinas A (una enterotoxina), la toxina B (una citotoxina) y la toxina binaria (13).

La ICD ocasiona, principalmente, úlceras pseudomembranosas y disbiosis, debido a un sobrecrecimiento de esta 
bacteria a nivel gastrointestinal, inducido por antibióticos como el metronidazol, la vancomicina y, más recientemente, la fidaxomicina o la rifaximina $(14,15)$. Estas terapias desencadenan eventos adversos como daño y muerte de la microbiota gastrointestinal humana, por lo que el TMF se considera una alternativa para corregir el desequilibrio subyacente en esta patogénesis y proporcionar a los pacientes enfermos una microbiota con alto grado homeostasis estructural y funcional, derivada de un donante adecuado (12).

En este sentido, Clostridioides difficile es la causa principal de diarrea asociada a los antibióticos, principalmente en pacientes hospitalizados en el mundo occidental, y se asocia con una alta morbimortalidad, así como con el uso de recursos sanitarios. Los aislados clínicos de Clostridioides difficile toxigénico son genéticamente diversos y se ha informado que algunos ribotipos hipervirulentos, como el 027, generan brotes en instalaciones de atención médica (16). Las manifestaciones clínicas de la ICD varían desde diarrea autolimitada, diarrea profusa, hasta colitis pseudomembranosa, íleo severo, megacolon tóxico, peritonitis e incluso choque o fallas orgánicas (13).

Asimismo, un número significativo de pacientes no responden al tratamiento inicial o sufren una recurrencia (2-38\%) en las primeras 8 semanas (17). La ICD recurrente se define como aquel episodio que ocurre hasta 8 semanas luego del inicio de una ICD previa, cuyos síntomas se hayan resuelto (18).

Es importante conocer la composición de la microbiota de los pacientes antes y después del trasplante, a fin de poder identificar los cambios generados (19). El TMF representa una terapia antibacteriana de muy bajo costo y es la más prometedora para el tratamiento de pacientes con ICD recurrente o refractaria al tratamiento con antibióticos. En efecto, se ha demostrado la superioridad del TMF en múltiples series de casos. Recientemente, en ensayos clínicos aleatorios prospectivos experimentales, se observó una resolución de la infección en más del 90 \% de los pacientes $(20)$.

\section{ABORDAJE HISTÓRICO DEL TMF}

Aunque parezca extraño, el TMF no es un concepto terapéutico nuevo. Existen datos muy antiguos que se refieren a él de manera más o menos directa. Por ejemplo, en el siglo IV, durante la dinastía china Dong Jin, el médico Ge Hong describió con buenos resultados la administración oral de una suspensión preparada a base de heces humanas en pacientes con intoxicación alimentaria o diarrea severa (21). Más tarde, Li Shizhen utilizó diversos preparados fecales para tratar todo tipo de males digestivos como diarreas, vómitos, dolor, fiebre o estreñimiento, a lo que llamó sopa amarilla. De igual manera, se destaca la ingesta de heces para aliviar ciertos padecimientos intestinales del ganado, descrita en el siglo XVII por Fabrizio d'Aquapendente $(20,22)$.

La ICD fue descrita por primera vez en 1978. Desde entonces ha sido señalada como la primera causa de diarrea intrahospitalaria y el principal origen identificable de diarrea asociada al uso indiscriminado de antibióticos (23). Durante la Segunda Guerra Mundial, los beduinos en el desierto norteafricano indicaban a los soldados la ingesta de heces de dromedario para tratar la disentería y otras enfermedades producidas por Clostridioides difficile.

El éxito del TMF en la medicina moderna fue reseñado por primera vez por Eiseman y colaboradores, en 1958. Estos investigadores administraron microbiota en enemas a pacientes con colitis pseudomembranosa (22), con el objetivo de desplazar los microbios patógenos del intestino, mediante el restablecimiento de una comunidad microbiana saludable. Con ello buscaron generar resultados eficientes para tratar la ICD (24). Dicho abordaje de la enfermedad, que desafió conceptualmente la visión vigente en aquella época de la microbiota como un elemento nocivo, cayó en el olvido de la comunidad científica durante más de medio siglo.

A partir de la última década, el TMF se ha posicionado como una de las terapias con mayor interés teórico y práctico en el ámbito de la gastroenterología, los procesos autoinmunitarios y las enfermedades metabólicas (17). Desde entonces, en la medicina moderna, los primeros TMF exitosos fueron reportados en 1958 por Eiseman y sus compañeros de trabajo, que trataron a 4 pacientes con colitis pseudomembranosa, causada por Clostridioides diffcile, anteriormente llamada Clostridium difficile.

Es así como, desde entonces, se han informado de manera constante las tasas de resolución del 70 al $90 \%$, después de un TMF, para la infección recurrente de ICD, tanto en los estudios observacionales como en los ensayos aleatorios (25). De igual forma, en 2013, Van Nood y colaboradores realizaron una publicación sobre su primer ensayo clínico aleatorizado, controlado y abierto, que examinó las ventajas terapéuticas del TMF en comparación con el tratamiento con vancomicina (26).

\section{PROCEDIMIENTO DEL TMF}

La selección y el cribado del donante en el TMF no se han estandarizado, por lo que los criterios en los diversos estudios son variables. Los tipos de donantes seleccionados pueden clasificarse en cuanto a su relación con el receptor en 4 grupos: familiares de sangre (54\%), individuos con contacto íntimo con el paciente (marido, esposa o pareja) (8 $\%)$, voluntarios sanos sin relación con el receptor (25\%) y donante no especificado (12\%) (22). Aunque se dice que el TMF es un procedimiento seguro, confiable y que no mues- 
tra ningún efecto adverso hasta el momento, a nivel teórico se habla acerca de riesgos que se pueden generar durante su realización, tales como la trasmisión de agentes infecciosos que desencadenan enfermedades a los receptores (13).

Por tanto, para evitar estas alteraciones, en primer lugar se recomienda realizar una serie de exámenes al donante como analítica sanguínea: hemograma y perfil viral (inmunoglobulina $\mathrm{M}$ [IgM] anti virus de la hepatitis A [VHA], antígeno de superficie [HBsAg]), inmunoglobulina G ( $\operatorname{IgG}$ ) e IgM anti virus de la hepatitis C (VHC), IgM anti virus de la hepatitis B (VHB), IgG anti VHC, virus de inmunodeficiencia humana (VIH) (1 y 2), anti virus linfotrópico humano (Human T-Lymphotropic Virus, HTLV) y sífilis (reagina plasmática rápida $[\mathrm{RPR}]$ y prueba de absorción de anticuerpos treponémicos fluorescentes [Fluorescent Treponemal Antibody-Absorption, FTA-ABS]).

Del mismo modo, es necesario realizar una prueba de reacción de la polimerasa en cadena (Polymerase Chain Reaction, PCR) en las heces para identificar microorganismos enteropatógenos y toxinas de Clostridioides difficile, así como un coprológico seriado (Giardia sp., Cryptosporidium sp., Cyclospora sp. e Isospora sp.) (27) y cuestionarios a fin de detectar conductas de riesgo (17).

Asimismo, se efectúa la selección del donante, considerando factores como su comportamiento sexual, transfusiones de sangre, su historial de viajes, antecedente de cirugía mayor en el aparato digestivo (se excluye la apendicectomía) (23), proceso oncológico activo o historia de cáncer en los últimos 10 años, enfermedad inflamatoria intestinal o dispepsia funcional (23) y otros aspectos que aumenten el riesgo de padecer una enfermedad transmisible (17).

Con respecto a la preparación del receptor, el acondicionamiento del colon parece reducir la densidad de bacterias como Clostridium difficile e incluso de sus esporas inactivas. Por ello, aunque su relación directa con la eficacia del TMF no ha sido demostrada, se recomienda el uso de soluciones evacuantes el día previo al procedimiento en aquellos pacientes cuya situación clínica lo permita, independientemente de la vía elegida para realizarlo (22).

Una vez se concluye la amplia selección y revisión de donantes (potenciales), se reciben las heces, las cuales deben procesarse lo más pronto posible (dentro de las primeras $6 \mathrm{~h}$ ) para mantener la viabilidad de la microbiota del donante. Se agrega un crioprotector a la suspensión fecal a fin de permitir un adecuado almacenamiento a $-80{ }^{\circ} \mathrm{C}$. Además, se debe almacenar una parte alícuota de cada donación para un posible análisis en caso de un evento adverso grave (28).

De la misma forma, la suspensión de heces del donante se puede mantener a una temperatura ambiente por hasta $3 \mathrm{~h}$, o refrigerada a $4{ }^{\circ} \mathrm{C}$ durante $6 \mathrm{~h}$ (29). Con ello se demuestra que el almacenamiento con mejor viabilidad se observa al suministrar glicerol, debido a que este favorece la viabilidad de la microbiota fecal. En efecto, se ha descubierto que los aislamientos de Escherichia coli son viables (y en proporción similares a las muestras frescas), luego de 1 año de almacenamiento congelado de las heces de lactantes $\mathrm{y}$ terneros, en $10 \%$ de caldo de glicerol a $-70^{\circ} \mathrm{C}$ (30).

Entre tanto, la ruta de administración preferida para el TMF sigue siendo un tema de discusión. En la actualidad, se han establecido distintos procedimientos para la realización del TMF, y las vías utilizadas han sido la digestiva superior y la digestiva inferior. Esta elección varía según la situación clínica del paciente (31).

El uso del tubo digestivo superior por vía nasogástrica o nasoduodenal es posible, fácil, de menor costo y supone un menor riesgo de perforación intestinal en comparación con la colonoscopia. Sin embargo, tiene la desventaja de que puede favorecer el sobrecrecimiento bacteriano en el intestino delgado. Además, es factible que no alcance los sitios distales con mayor afección y puede producir síntomas desagradables para el paciente, como el reflujo o la distensión abdominal $(32,33)$.

Asimismo, el TMF por vía superior puede realizarse mediante cápsulas ingeridas (34). De hecho, un estudio reciente mostró altas tasas de curación en pacientes tratados con TMF por cápsula oral, un método que puede disminuir la incomodidad del enfermo. Sin embargo, esta práctica requiere la ingestión de grandes cantidades de cápsulas, las cuales no están fácilmente disponibles (30).

De otro lado, la administración a través del tubo digestivo inferior se realiza mediante una colonoscopia (la vía de elección) o mediante enemas. La colonoscopia permite la visualización directa de la mucosa, aunque puede asociarse con un mayor riesgo de perforación, sobre todo en pacientes con megacolon tóxico $(32,35)$.

\section{EFECTIVIDAD Y DESVENTAJAS DEL TMF}

La alta efectividad del TMF, con una respuesta $>80 \%$ en revisiones sistemáticas en el escenario de la ICD recurrente, ha estimulado el interés de esta terapia tanto en pacientes, médicos e investigadores como en la industria farmacéutica. Así pues, el TMF ya no es considerado un recurso excepcional en la ICD recurrente y cada vez más se practica de forma común (36).

Ahora bien, las desventajas de esta terapia se pueden clasificar de acuerdo con su aparición a corto y largo plazo. Las primeras se relacionan con la manifestación de dolor abdominal, distensión, flatulencia, diarrea y fiebre, mientras que las segundas incluyen síntomas como perforación, hemorragia y depresión cardiorrespiratoria (37). También se han reportado casos de transmisión de agentes infecciosos y bacteriemia. 
Además, los efectos a largo plazo tienen relación con la modulación de ciertas enfermedades como obesidad, diabetes mellitus, ateroesclerosis, hígado graso, enfermedad inflamatoria intestinal, síndrome de intestino irritable, asma y autismo $(31,34)$.
A continuación, se identifican los principales estudios de revisión sistemática y de literatura, con hallazgos representativos en cuanto a la efectividad y los eventos adversos presentados luego de un TMF (Tabla 1) (17, 34, 38-41):

Tabla 1. Eficacia frente a eventos adversos del TMF
Año País
Tipo de estudio
Resultados (38) estudios con 611 pacientes.
2015 China Revisión sistemática que incluyó 18

Se reportó una tasa de curación primaria del $91,2 \%$. Se registraron 6 muertes por ICD. En total, se informaron 38 muertes en 7 estudios; entre ellas, 6 fueron asociadas con la ICD recurrente o con la ICD agresiva por la cepa 027 de $C$. difficile. Entre tanto, 3 muertes se asociaron con enfermedades infecciosas no relacionadas, como neumonía y peritonitis.

Cerca de 69 casos de síntomas gastrointestinales fueron reportados en 11 estudios. De estos síntomas, los más comunes fueron flatulencias, dolor abdominal, calambres y diarrea. Casi todos fueron de corta duración, moderados y manejables.

Otros efectos adversos incluyeron fiebre autolimitada y enfermedades emergentes como septicemia, neumonía y peritonitis (todas infecciosas). Se identificó como factor de riesgo para tener más complicaciones una edad mayor a 65 años.
2016 Inglaterra Revisión de literatura que incluyó 109 estudios con 1555 pacientes.

Se encontraron eventos adversos leves y autolimitados, de naturaleza gastrointestinal, como flatulencias, diarrea, dolor y distensión abdominal, constipación y náuseas. En algunos casos de complicaciones graves, no se estableció una asociación creíble, debido a la falta de datos controlados.

Las complicaciones graves fueron 3 muertes causadas por bacteriemia, insuficiencia respiratoria y regurgitación de material feculento.

2017 Inglaterra Se incluyeron 37 estudios: 7 ensayos controlados aleatorios y 30 series de casos, con un total de 1973 pacientes.

La resolución clínica en todos los estudios fue del $92 \%$. El TMF fue más efectivo que la terapia con vancomicina para la ICD. La diarrea leve, los calambres transitorios, el estreñimiento a largo plazo y la flatulencia fueron los eventos adversos leves más frecuentes.

Hubo 50 muertes reportadas en los estudios revisados. Sin embargo, casi todas se debieron a enfermedades críticas de pacientes de edad avanzada. Se presentó una muerte como resultado de aspiración en el momento de la sedación en la colonoscopia para administrar el TMF.

Además, 2 pacientes con diarrea recurrente murieron después del TMF, por complicaciones de íleo y perforación colónica. Es de destacar que una serie de casos de TMF, en 80 pacientes inmunocomprometidos, con seguimiento de 3 meses, no informó ningún efecto adverso grave.

Solo se informaron 3 eventos adversos importantes y no se produjeron muertes directamente relacionadas con el TMF. En total, 285 pacientes respondieron al primer tratamiento, sin recurrencia durante el seguimiento especificado, mientras que 42 fueron sometidos a un segundo tratamiento, con resolución de los síntomas en 28 de ellos.

Se informó que al menos 5 pacientes se sometieron a un tercer tratamiento, con resolución en 3 de ellos. Solo un paciente informó haber recibido 4 tratamientos, sin resolución a largo plazo de los síntomas.

Con respecto a la eficacia, se demostró que el TMF encapsulado es seguro y rentable para el tratamiento y la prevención de la ICD recurrente. 


\begin{tabular}{|c|c|c|c|}
\hline Año & País & Tipo de estudio & Resultados \\
\hline $\begin{array}{l}2018 \\
(41)\end{array}$ & $\begin{array}{l}\text { Estados } \\
\text { Unidos }\end{array}$ & $\begin{array}{l}\text { Se incluyeron } 44 \text { estudios, ninguno de } \\
\text { los cuales fueron diseños aleatorios. } \\
\text { Se estudió un total de } 303 \text { pacientes } \\
\text { inmunocomprometidos que consumían } \\
\text { medicamentos inmunosupresores. } \\
\text { Entre ellos, se incluyeron pacientes, } \\
\text { de todas las edades, con VIH, } \\
\text { síndromes de inmunodeficiencia } \\
\text { heredada o primaria, cáncer sometido } \\
\text { a quimioterapia o trasplante de órganos } \\
\text { (incluido el trasplante de médula ósea). }\end{array}$ & $\begin{array}{l}\text { El } 76 \% \text { de los pacientes recibieron el TMF mediante una colonoscopia. De los } 234 \text { con } \\
\text { resultados de seguimiento reportados, } 207 \text { ( } 87 \% \text { ) informaron la resolución después } \\
\text { del primer tratamiento, con un } 93 \% \text { de efectividad, lo cual indicó el éxito después de } \\
\text { múltiples tratamientos. } \\
\text { Se reportaron } 2 \text { muertes, } 2 \text { colectomías, } 5 \text { infecciones relacionadas con el } \\
\text { tratamiento y } 10 \text { hospitalizaciones posteriores. Se concluyó que, en los pacientes } \\
\text { inmunocomprometidos, el TMF parece tener datos comparables de eficacia y } \\
\text { seguridad similares a los de pacientes inmunocompetentes. } \\
\text { Sin embargo, debido a la heterogeneidad del subtipo de inmunosupresión, no se } \\
\text { puede llegar a una conclusión sólida a cerca de cualquier estado inmunocomprometido } \\
\text { específico, o de una combinación con respecto a la respuesta al TMF. Es necesario } \\
\text { hacer ensayos aleatorios adicionales. }\end{array}$ \\
\hline $\begin{array}{l}2018 \\
(17)\end{array}$ & Chile & $\begin{array}{l}\text { Estudio observacionales de pacientes } \\
\text { con ICD recurrente: } 2013-2017 .\end{array}$ & $\begin{array}{l}\text { El TMF se realizó en } 8 \text { pacientes con ICD recurrente; } 6 \text { de ellos eran mujeres. La mediana } \\
\text { de edad fue de } 48 \text { años. La efectividad del TMF fue del } 100 \% \text { y todos los pacientes } \\
\text { presentaron una respuesta clínica con deposiciones formadas dentro de la semana. } \\
\text { Ningún paciente presentó un nuevo episodio de diarrea dentro de los } 6 \text { meses } \\
\text { posteriores al TMF. }\end{array}$ \\
\hline
\end{tabular}

TMF: trasplante de microbiota fecal; ICD: infección por Clostridioides difficile; C. difficile: Clostridioides difficile. Tomada de las referencias 17, 34, 38-41.

De acuerdo con lo anterior, se identificó que el TMF parece ser efectivo y se convierte en una estrategia aparentemente segura en el tratamiento de la ICD recurrente y refractaria. En efecto, está documentado que la tasa de resolución de la ICD es directamente proporcional al volumen de heces infundido. A su vez, la recurrencia del cuadro clínico se asocia con el peso de heces usado en el trasplante (17). La eficacia es similar en estudios controlados y no controlados. Asimismo, se han notificado más eventos adversos en el TMF para el tratamiento de la enfermedad inflamatoria intestinal que para la ICD. Además, no existen ensayos controlados con placebo verdaderos que investiguen la eficacia del TMF.

\section{OTROS USOS DEL TMF}

En la actualidad, el TMF se está convirtiendo en una opción de tratamiento de alta efectividad para las ICD y otras disbiosis, así como para encefalopatías hepáticas, el síndrome de intestino irritable y la enfermedad inflamatoria intestinal. Estos trastornos metabólicos afectan en gran medida a los pacientes (42). Hoy se habla que el TMF, incluso, puede tener un papel importante en el tratamiento de la obesidad (43), la psoriasis, el cáncer y la enfermedad de Parkinson (44).

Dentro de ese contexto, están en marcha ensayos clínicos para el uso del TMF en el tratamiento de la enfermedad infla- matoria intestinal, las encefalopatías hepáticas, la colangitis esclerosante primaria, la pancreatitis aguda, el estreñimiento, la esteatorrea, la erradicación de bacterias multirresistentes en portadores fecales, el VIH y la epilepsia (32).

Entre tanto, en pacientes con trastorno del espectro autista se han observado alteraciones gastrointestinales como disbiosis, con la presencia de infección por el género Clostridium, incluidas cepas de Clostridioides difficile. Sin embargo, esta no es la única cepa susceptible de cursar con alteraciones del comportamiento en niños autistas. También se han descrito otros microorganismos como Candida spp. Así pues, se considera que el TMF regenera la microbiota intestinal, a partir de la producción de abundante diversidad de microrganismos bacterianos (45).

Por otro lado, se realizó un estudio abierto de pequeño ensayo clínico, fase I, en 18 niños entre 6 y 17 años, que consistió en el tratamiento combinado de antibiótico por 2 semanas, limpieza intestinal y luego TMF con dosis inicial alta, seguido de dosis diarias de mantenimiento más bajas durante 7-8 semanas. En él se evidenció un cambio significativo relativo a la abundancia de Bifido bacterium spp., Prevotella spp. y Desulfovibrio spp. en el control de la composición de la microbiota. Se observó una mejoría de los síntomas gastrointestinales, así como en el comportamiento del lenguaje ( $25 \%$ ), la interacción social, la conducta repetitiva, la hiperactividad y la irritabilidad, lo cual se mantuvo durante 8 semanas (46). 
Estudios recientes relacionan la microbiota intestinal con la fisiopatología de la obesidad. El uso del TMF en la obesidad y la diabetes mellitus tipo 2 se fundamenta en la composición de la microbiota intestinal, ya que esta varía significativamente entre los sujetos obesos y delgados. Se ha publicado, además, que el grupo que recibe TMF tiene un significativo aumento de la sensibilidad a la insulina postrasplante (2). Los resultados de las investigaciones de pacientes con síndrome metabólico o diabetes tipo 2 son alentadores a la luz de la pandemia de obesidad en expansión, y requieren una mayor exploración. También se necesitan estudios adicionales para determinar los efectos de las alteraciones de la microbiota en el crecimiento tumoral y terapias contra algunos tipos de cáncer (47).

\section{CONCLUSIONES}

Si bien existen diversas técnicas para realizar el TMF de acuerdo con diversos protocolos, el procedimiento en sí mismo se considera seguro. Además, se observa la necesi- dad de estandarizar y aleatorizar ensayos controlados para calificar y cuantificar los riesgos del TMF. Este procedimiento terapéutico, económicamente accesible, sencillo y validado con evidencia científica, abre el panorama de investigación en otras enfermedades del ser humano, tanto digestivas como extradigestivas, que representan altos costos sanitarios, reingresos, estancias hospitalarias prolongadas y morbimortalidad elevada.

\section{Agradecimientos}

Ninguno declarado por los autores.

\section{Conflicto de interés}

Ninguno declarado por los autores.

\section{Fuente de financiación}

Ninguna declarada por los autores.

\section{REFERENCIAS}

1. Stedman A, Nigro G, Sansonetti PJ. Le dialogue microbiote-cellules souches: un élément clé pour la régénération intestinale. Med Sci. 2016;32(11):983-990. https://doi.org/10.1051/medsci/20163211014

2. Rodríguez de Santiago E, García A, Ferre C, Aguilera L, López A. Trasplante de microbiota fecal: indicaciones, metodología y perspectivas futuras. Rev Argent Coloproct. 2015;26(4):225-234.

3. Allegretti JR, Mullish BH, Kelly C, Fischer M. The evolution of the use of faecal microbiota transplantation and emerging therapeutic indications. Lancet. 2019;394(10196):420-431. http://doi.org/10.1016/S0140-6736(19)31266-8

4. Tavoukjian V. Faecal microbiota transplantation for the decolonization of antibiotic-resistant bacteria in the gut: a systematic review and meta-analysis. J Hosp Infect. 2019;102(2):174-188. http://doi.org/10.1016/j.jhin.2019.03.010

5. Leszczyszyn JJ, Radomski M, Leszczyszyn AM. Intestinal microbiota transplant - current state of knowledge. Reumatologia. 2016;54(1):24-28. http://doi.org/10.5114/reum.2016.58758

6. Human Microbiome Project Consortium. Structure, function and diversity of the healthy human microbiome. Nature. 2012;486(7402):207-214. http://doi.org/10.1038/nature 11234

7. Moore-Connors JM, Dunn KA, Bielawski JP, Van Limbergen J. Novel Strategies for Applied Metagenomics.
Inflamm Bowel Dis. 2016;22(3):709-718. http://doi.org/10.1097/MIB.0000000000000717

8. Ho J, Nicolucci AC, Virtanen H, Schick A, Meddings J, Reimer RA, Huang C. Effect of Prebiotic on Microbiota, Intestinal Permeability, and Glycemic Control in Children With Type 1 Diabetes. J Clin Endocrinol Metab. 2019; 104(10):4427-4440. http://doi.org/10.1210/jc.2019-00481

9. de Goffau MC, Luopajärvi K, Knip M, Ilonen J, Ruohtula T, Härkönen T, Orivuori L, Hakala S, Welling GW, Harmsen HJ, Vaarala O. Fecal microbiota composition differs between children with $\beta$-cell autoimmunity and those without. Diabetes. 2013;62(4):1238-44. http://doi.org/10.2337/db12-0526

10. Silva F, Gatica T, Pavez C. Etiología y fisiopatología de la enfermedad inflamatoria intestinal. Rev Med Clínica Las Condes. 2019;30(4):262-272. https://doi.org/10.1016/j.rmclc.2019.06.004

11. Icaza-Chávez ME. Microbiota intestinal en la salud y la enfermedad. Rev Gastroenterol Mex. 2013;78(4):240-248. http://doi.org/10.1016/j.rgmx.2013.04.004

12. Borody TJ, Paramsothy S, Agrawal G. Fecal microbiota transplantation: indications, methods, evidence, and future directions. Curr Gastroenterol Rep. 2013;15(8):337. http://doi.org/10.1007/s11894-013-0337-1

13. Lin TC, Hung YP, Ko WC, Ruan JW. Fecal microbiota transplantation for Clostridium difficile infection in Taiwan: Establishment and implementation. J Microbiol 
Immunol Infect. 2019;52(6):841-850.

http://doi.org/10.1016/j.jmii.2019.08.009

14. Huebner ES, Surawicz CM. Treatment of Recurrent Clostridium difficile Diarrhea. Gastroenterol Hepatol (N Y). 2006;2(3):203-208.

15. Sabah S. diarrea asociada a antibióticos. Rev Med Clínica Las Condes. 2015;26(5):687-695. https://doi.org/10.1016/j.rmclc.2015.09.011

16. Leffler DA, Lamont JT. Clostridium difficile infection. $\mathrm{N}$ Engl J Med. 2015;372(16):1539-1548. http://doi.org/10.1056/NEJMra1403772

17. López-Sanromán A, Rodríguez de Santiago E, Cobo Reinoso J, Del Campo Moreno R, Foruny Olcina JR, García Fernández S, García García de Paredes A, Aguilera Castro L, Ferre Aracil C, Albillos Martínez A. Results of the implementation of a multidisciplinary programme of faecal microbiota transplantation by colonoscopy for the treatment of recurrent Clostridium difficile infection. Gastroenterol Hepatol. 2017;40(9):605-614. http://doi.org/10.1016/j.gastrohep.2017.03.004

18. Cohen SH, Gerding DN, Johnson S, Kelly CP, Loo VG, McDonald LC, Pepin J, Wilcox MH; Society for Healthcare Epidemiology of America; Infectious Diseases Society of America. Clinical practice guidelines for Clostridium difficile infection in adults: 2010 update by the society for healthcare epidemiology of America (SHEA) and the infectious diseases society of America (IDSA). Infect Control Hosp Epidemiol. 2010;31(5):431-55. http://doi.org/10.1086/651706

19. Doré J, Simrén M, Buttle L, Guarner F. Hot topics in gut microbiota. United European Gastroenterol J. 2013;1(5):311-318. http://doi.org/10.1177/2050640613502477

20. Vindigni SM, Surawicz CM. Fecal Microbiota Transplantation. Gastroenterol Clin North Am. 2017;46(1):171-185. http://doi.org/10.1016/j.gtc.2016.09.012

21. Zamudio-Tiburcio Á, Bermúdez-Ruiz H, Lezama-Guzmán HR, Guevara-Ortigoza MDP, Islas-Solares E, Sosa-López FA. Breaking paradigms. Intestinal microbiota transplantation: Preliminar report. Cir Cir. 2017;85 Suppl 1:6-12. http://doi.org/10.1016/j.circir.2016.11.017

22. García A, Rodríguez de Santiago E, Aguilera L, Ferre C, López-Sanromán A. Trasplante de microbiota fecal. 2015;38(3):123-134. https://doi.org/10.1016/j.gastrohep.2014.07.010

23. Quera R, Ibáñez P, Simian D, Rivera D, Acuña G, Espinoza R. Fecal microbiota transplantation through colonoscopy for Clostridium difficile recurrent infection. Report of eight cases. Rev Med Chil. 2018;146(8):823-830. http://doi.org/10.4067/s0034-98872018000800823

24. Smith MB, Kelly C, Alm EJ. Policy: How to regulate faecal transplants. Nature. 2014;506(7488):290-291. http://doi.org/10.1038/506290a

25. Jørgensen SMD, Hvas CL, Dahlerup JF, Mikkelsen S, Ehlers L, Hammeken LH, Licht TR, Bahl MI, Erikstrup
C. Banking feces: a new frontier for public blood banks? Transfusion. 2019;59(9):2776-2782. http://doi.org/10.1111/trf.15422

26. Verbeke F, Janssens Y, Wynendaele E, De Spiegeleer B. Faecal microbiota transplantation: a regulatory hurdle?. BMC Gastroenterol. 2017;17(1):128. http://doi.org/10.1186/s12876-017-0687-5

27. Cammarota G, Ianiro G, Tilg H, Rajilić-Stojanović M, Kump P, Satokari R, Sokol H, Arkkila P, Pintus C, Hart A, Segal J, Aloi M, Masucci L, Molinaro A, Scaldaferri F, Gasbarrini G, Lopez-Sanroman A, Link A, de Groot P, de Vos WM, Högenauer C, Malfertheiner P, Mattila E, Milosavljević T, Nieuwdorp M, Sanguinetti M, Simren M, Gasbarrini A; European FMT Working Group. European consensus conference on faecal microbiota transplantation in clinical practice. Gut. 2017;66(4):569-580. http://doi.org/10.1136/gutjnl-2016-313017

28. Ooijevaar RE, Terveer EM, Verspaget HW, Kuijper EJ, Keller JJ. Clinical Application and Potential of Fecal Microbiota Transplantation. Annu Rev Med. 2019;70:335-351. http://doi.org/10.1146/annurev-med-111717-122956

29. Terveer EM, van Beurden YH, Goorhuis A, Seegers JFML, Bauer MP, van Nood E, Dijkgraaf MGW, Mulder CJJ, Vandenbroucke-Grauls CMJE, Verspaget HW, Keller JJ, Kuijper EJ. How to: Establish and run a stool bank. Clin Microbiol Infect. 2017;23(12):924-930. http://doi.org/10.1016/j.cmi.2017.05.015

30. Costello SP, Conlon MA, Vuaran MS, Roberts-Thomson IC, Andrews JM. Faecal microbiota transplant for recurrent Clostridium difficile infection using long-term frozen stool is effective: clinical efficacy and bacterial viability data. Aliment Pharmacol Ther. 2015;42(8):1011-1018. http://doi.org/10.1111/apt.13366

31. Castañeda C. Trasplante de microbiota fecal. Rev Cubana Pediatr. 2019;91(3):e829.

32. Núñez ME, Cebada M, Contreras B. Trasplante de microbiota fecal: protocolo de estandarización para la selección de donadores. An Med (Mex). 2017;62(2):114-119.

33. Shi YC, Yang YS. Fecal microbiota transplantation: Current status and challenges in China. JGH Open. 2018;2(4):114-116. http://doi.org/10.1002/jgh3.12071

34. Iqbal U, Anwar H, Karim MA. Safety and efficacy of encapsulated fecal microbiota transplantation for recurrent Clostridium difficile infection: a systematic review. Eur J Gastroenterol Hepatol. 2018;30(7):730-734. http://doi.org/10.1097/MEG.0000000000001147

35. Scaldaferri F, Pecere S, Petito V, Zambrano D, Fiore L, Lopetuso LR, Schiavoni E, Bruno G, Gerardi V, Laterza L, Pizzoferrato M, Ianiro G, Stojanovic J, Poscia A, Papa A, Paroni Sterbini F, Sanguinetti M, Masucci L, Cammarota G, Gasbarrini A. Efficacy and Mechanisms of Action of Fecal Microbiota Transplantation in Ulcerative Colitis: Pitfalls and Promises From a First Meta-Analysis. Transplant Proc. 2016;48(2):402-7. http://doi.org/10.1016/j.transproceed.2015.12.040 
36. Paknikar R, Pekow J. Fecal Microbiota Transplantation for the Management of Clostridium difficile Infection. Surg Infect (Larchmt). 2018;19(8):785-791. http://doi.org/10.1089/sur.2018.221

37. Wang S, Xu M, Wang W, Cao X, Piao M, Khan S, Yan F, Cao H, Wang B. Systematic Review: Adverse Events of Fecal Microbiota Transplantation. PLoS One. 2016;11(8):e0161174. http://doi.org/10.1371/journal.pone.0161174

38. Li YT, Cai HF, Wang ZH, Xu J, Fang JY. Systematic review with meta-analysis: long-term outcomes of faecal microbiota transplantation for Clostridium difficile infection. Aliment Pharmacol Ther. 2016;43(4):445-457. http://doi.org/10.1111/apt.13492

39. Baxter M, Colville A. Adverse events in faecal microbiota transplant: a review of the literature. J Hosp Infect. 2016;92(2):117-127. http://doi.org/10.1016/j.jhin.2015.10.024

40. Quraishi MN, Widlak M, Bhala N, Moore D, Price M, Sharma N, Iqbal TH. Systematic review with meta-analysis: the efficacy of faecal microbiota transplantation for the treatment of recurrent and refractory Clostridium difficile infection. Aliment Pharmacol Ther. 2017;46(5):479-493. http://doi.org/10.1111/apt.14201

41. Shogbesan O, Poudel DR, Victor S, Jehangir A, Fadahunsi O, Shogbesan G, Donato A. A Systematic Review of the Efficacy and Safety of Fecal Microbiota Transplant for Clostridium difficile Infection in Immunocompromised Patients. Can J Gastroenterol Hepatol. 2018;2018:1394379. http://doi.org/10.1155/2018/1394379

42. Sha S, Liang J, Chen M, Xu B, Liang C, Wei N, Wu K. Systematic review: faecal microbiota transplantation the- rapy for digestive and nondigestive disorders in adults and children. Aliment Pharmacol Ther. 2014;39(10):1003-32. http://doi.org/10.1111/apt.12699

43. Gundling F, Roggenbrod S, Schleifer S, Sohn M, Schepp W. Patient perception and approval of faecal microbiota transplantation (FMT) as an alternative treatment option for obesity. Obes Sci Pract. 2018;5(1):68-74. http://doi.org/10.1002/osp4.302

44. Sampson TR, Debelius JW, Thron T, Janssen S, Shastri GG, Ilhan ZE, Challis C, Schretter CE, Rocha S, Gradinaru V, Chesselet MF, Keshavarzian A, Shannon KM, KrajmalnikBrown R, Wittung-Stafshede P, Knight R, Mazmanian SK. Gut Microbiota Regulate Motor Deficits and Neuroinflammation in a Model of Parkinson's Disease. Cell. 2016;167(6):1469-1480.e12. http://doi.org/10.1016/j.cell.2016.11.018

45. Castañeda CD. Microbiota intestinal, probióticos y prebióticos. Enferm Inv (Ambato). 2017;2(4):156-160. https://doi.org/10.29033/ei.v2n4.2017.07

46. Kang DW, Adams JB, Gregory AC, Borody T, Chittick L, Fasano A, Khoruts A, Geis E, Maldonado J, McDonoughMeans S, Pollard EL, Roux S, Sadowsky MJ, Lipson KS, Sullivan MB, Caporaso JG, Krajmalnik-Brown R. Microbiota Transfer Therapy alters gut ecosystem and improves gastrointestinal and autism symptoms: an openlabel study. Microbiome. 2017;5(1):10. http://doi.org/10.1186/s40168-016-0225-7

47. D’Haens GR, Jobin C. Fecal Microbial Transplantation for Diseases Beyond Recurrent Clostridium Difficile Infection. Gastroenterology. 2019;157(3):624-636. http://doi.org/10.1053/j.gastro.2019.04.053 